\title{
Evaluation of Six Split-thickness Skin Graft Donor-site Dressing Materials in a Swine Model
}

\author{
Pamela C. Masella, DO* \\ Eric M. Balent, MD* \\ Terri L. Carlson, DO* \\ Karen W. Lee, BS† \\ Lisa M. Pierce, DSc $†$
}

Background: Numerous dressings for split-thickness skin graft donor sites are commercially available with no conclusive evidence-based consensus regarding the optimal dressing choice. This study was conducted to identify which of 5 commonly used materials promotes wound healing most effectively for use on split-thickness donor sites in comparison with our standard dressing, Xeroform (petrolatum gauze).

Methods: Twenty-four partial-thickness wounds were created on the backs of 4 pigs using a dermatome. Wounds ( $n=4$ per dressing type per pig) were treated with Xeroform, Opsite (polyurethane film), Kaltostat (calcium sodium alginate), DuoDERM (hydrocolloid), Aquacel (hydrofiber), and Mepilex (silicone foam). Full-thickness skin samples were excised at 3 or 5 days and evaluated histologically for reepithelialization and inflammation. Comparisons also included incidence of infection, ease of use, and cost analyses.

Results: DuoDERM elicited the greatest percent reepithelialization $(81 \%)$ and Mepilex the lowest $(33 \%)$ after 3 days $(P=0.004)$. All dressings demonstrated complete reepithelialization except Mepilex (85\%) at 5 days. There were no infections and inflammation was mild among all treatments. Mepilex was easiest to use, whereas Aquacel, Kaltostat, and Opsite were most difficult $(P=0.03)$. Xeroform was most cost-effective and Aquacel most expensive. Combined scoring revealed DuoDERM $=$ Xeroform $>$ Opsite $=$ Mepilex $>$ Kaltostat $>$ Aquacel.

Conclusions: DuoDERM and Xeroform were most effective overall. DuoDERM tended to outperform all dressings in reepithelialization at 3 days, while Xeroform was least expensive, easy to use, and demonstrated rapid reepithelialization. These findings suggest that Xeroform may be preferred for use on large donor-site areas. DuoDERM may be more appropriate for small donor sites when healing time is a priority. (Plast Reconstr Surg Glob Open 2013;1:e84; doi: 10.1097/GOX.0000000000000031; Published online 19 December 2013) plit-thickness skin grafting is a widely used reconstructive technique for the replacement of damaged or missing skin caused by burns, trauma, surgical resection for cancer, and chronic wounds. ${ }^{1}$ Split-thickness autografts are harvested by excising the epidermis and part of the dermis, leaving

From the *Department of General Surgery, Tripler Army Medical Center, Honolulu, Hawaii; and †Department of Clinical Investigation, Tripler Army Medical Center, Honolulu, Hawaii.

Received for publication June 18, 2013; accepted November 7, 2013.

PresentedatPlasticSurgery TheMeeting2013, October 11-15, 2013, San Diego, CA. a donor-site wound that can vary in thickness. ${ }^{1}$ Donorsite wounds generally heal by reepithelialization in 7-14 days, after which they may be used repeatedly when a large defect necessitating repair exists, such as an extensive burn..$^{2-5}$ Therefore, proper wound care of the donor site is critical to reduce time to complete

Copyright (C) 2013 The Authors. Published by Lippincott Williams E Wilkins on behalf of The American Society of Plastic Surgeons. PRS Global Open is a publication of the American Society of Plastic Surgeons. This is an open-access article distributed under the terms of the Creative Commons AttributionNonCommercial-NoDerivatives 3.0 License, where it is permissible to download and share the work provided it is properly cited. The work cannot be changed in any way or used commercially. DOI: $10.1097 /$ GOX.0000000000000031 
reepithelialization and to prevent significant morbidity resulting from delayed healing, infection, or conversion of the donor site to a full-thickness wound. ${ }^{6-9}$

Optimal donor-site dressings promote wound healing by preventing dessication, removing excess exudate, allowing gaseous exchange, and accelerating reepithelialization while being comfortable for the patient, resistant to infection, easily applied, and cost-effective. ${ }^{7-14}$ Although petrolatum fine-mesh gauze impregnated with the antiseptic bismuth tribromophenate (Xeroform) is the preferred dressing at our institution and in some burn centers around the world, ${ }^{15}$ we question whether a more effective dressing is available. Review of the literature has shown that impregnated fine-mesh gauze, calcium alginate, and polyurethane film are among the most commonly used dressings; however, newer materials such as hydrofibers, hydrocolloids, and foams are becoming more popular for use on split-thickness donor sites. . $^{3,8,15,16}$

Results of national and worldwide surveys indicate that practitioners often use the dressing they are most familiar with, regardless of performance. ${ }^{3,15,16}$ This is due, in part, to an overabundance of choices and a lack of consistent clinical evidence to support alternative materials., ${ }^{3,12,15,16}$ To date, 4 systematic reviews on the most effective donor-site dressings have been completed..$^{7,10,17,18}$ These reviews were based mainly on small trials comparing a limited number of dressing types. Aggregate clinical evidence suggests that hydrocolloids and films may be superior to other materials, to include Xeroform; however, the authors agree that more conclusive evidence is needed. ${ }^{7,10,17,18}$ The largest randomized clinical trial evaluating 6 commonly used dressings was published in 2013 by the Recognizing Effective Materials By Randomizing and Assessing New Donorsite Treatments (REMBRANDT) study group. ${ }^{8}$ The authors reported that the use of hydrocolloid dressings resulted in the fastest healing of donor-site wounds, while gauze dressings were accompanied by a higher infection rate. ${ }^{8}$ Importantly, this randomized trial did not evaluate Xeroform. ${ }^{8}$

Disclosure: The authors have no financial interest to declare in relation to the content of this article. This study was supported by intramural funds from the Department of Clinical Investigation, Tripler Army Medical Center. The views expressed in this article are those of the authors and do not reflect the official policy or position of the Department of the Army, Department of Defense, or the US Government. The Article Processing Charge was paid for by the authors.
Although human studies are the most accurate way to determine the clinical effectiveness of donor-site dressings, it is often difficult to obtain sufficient numbers of similar wounds for randomized trials, thus limiting the number of materials compared. In addition, objective measurements of wound healing are restricted due to the need for biopsies obtained for histologic analysis. Animal models are thus a viable alternative to provide additional evidence to support the use of various dressings, with the pig being the most suitable animal model available because porcine skin is similar to that of humans in epidermal thickness, composition, vascularization, and healing. ${ }^{19}$ In this study, we used a swine model to determine which of 5 commonly used dressing materials, including polyurethane film (Opsite), calcium alginate (Kaltostat), hydrofiber (Aquacel), hydrocolloid (DuoDERM), and silicone-faced polyurethane foam (Mepilex), promotes wound healing most effectively for use on split-thickness skin graft donor sites when compared with our standard dressing, Xeroform. Parameters examined included reepithelialization, inflammation, prevention of infection, ease of use (application and wound care), and cost.

\section{MATERIALS AND METHODS}

\section{Animals}

Four female Yorkshire pigs weighing approximately $25 \mathrm{~kg}$ were obtained from a local farm. The study protocol was approved by the Institutional Animal Care and Use Committee at Tripler Army Medical Center. Investigators complied with the policies as prescribed in the U.S. Department of Agriculture Animal Welfare Act and the National Research Council's Guide for the Care and Use of Laboratory Animals. Facilities are fully accredited by the Association for Assessment and Accreditation of Laboratory Animal Care International.

\section{Treatments and Study Design}

Six dressings were evaluated in this study including petrolatum gauze (Xeroform; Covidien, Mansfield, Mass.), polyurethane film (Opsite Flexigrid; Smith \& Nephew, London, UK), calcium sodium alginate (Kaltostat; ConvaTec, Skillman, N.J.), hydrocolloid (DuoDERM Extra Thin; ConvaTec), hydrofiber (Aquacel; ConvaTec), and silicone-faced polyurethane foam (Mepilex; Mölnlycke Healthcare, Göteborg, Sweden). Twenty-four partial-thickness wounds (approximately $4 \times 2 \mathrm{~cm}$ ) were created on the back of each pig, with 4 wounds treated with the same dressing type (Fig. 1). Wounds treated with different materials were sepa- 

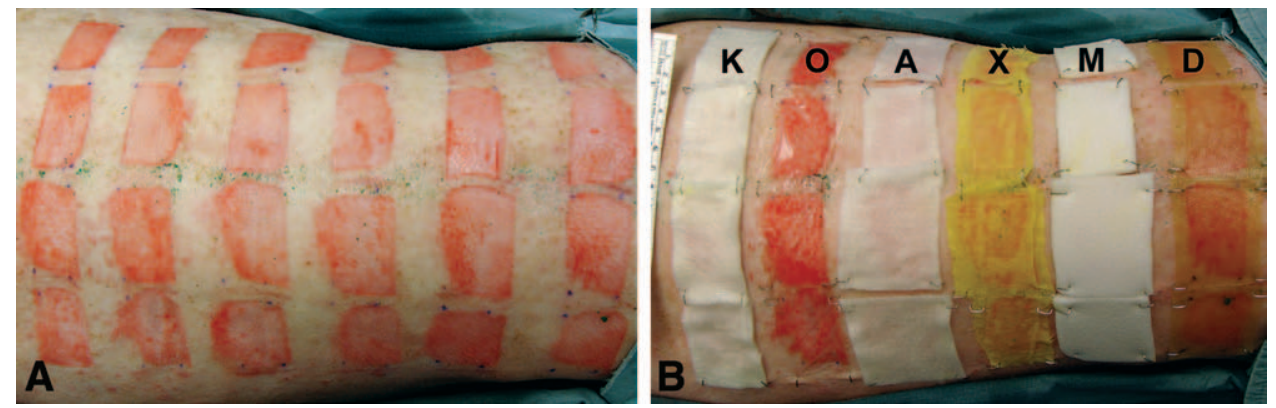

Fig. 1. Example of wound treatment arrangement on day 0. Twenty-four split-thickness wounds per pig were created using an electric dermatome set at 0.015 inches in depth (A). Four wounds each were treated (B) with Kaltostat (K), Opsite (O), Aquacel (A), Xeroform (X), Mepilex (M), and DuoDERM (D).

rated by approximately $3 \mathrm{~cm}$ and the arrangement of dressings was randomized before each surgery to minimize cross-reactivity or interference between dressings. Full-thickness skin samples were excised at 3 days (3 pigs) or 5 days ( 1 pig) and evaluated histologically for reepithelialization and inflammation. Dressings were also assessed for incidence of infection, ease of use, and cost.

\section{Surgery and Wound Care}

One day before surgery, pigs were administered a fentanyl patch $(50 \mu \mathrm{g} / \mathrm{h})$, which was replaced after 72 hours. Anesthesia was induced with an intramuscular injection of ketamine $(33 \mathrm{mg} / \mathrm{kg})$ and midazolam $(0.5 \mathrm{mg} / \mathrm{kg})$ and, after intubation, was maintained with inhaled $2 \%$ isoflurane. Pigs were given one dose of cefazolin $(1 \mathrm{~g})$ intravenously before the procedure. The back of each pig was shaved and proposed wound areas $(4 \times 2 \mathrm{~cm})$ were marked with a skin marker and template. The surgical site was then prepped with chlorhexidine, draped in a standard sterile fashion, and sterile mineral oil was applied to the skin. Twentyfour partial-thickness wounds were created using an electric dermatome (Model B Padgett Electro Dermatome, Integra Life Sciences, Cincinnati, Ohio) set at 0.015 inches in depth. The skin was discarded, and hemostasis from the donor sites was achieved with saline-soaked gauze and pressure. Dressings were cut into approximately $5 \times 3 \mathrm{~cm}$ pieces, applied to the randomly assigned wounds, and secured in place using a sterile skin stapler. These were then covered with layers of sterile gauze, abdominal pads, Chux, tape, and a goat tube (Sullivan Supply, Tex.) for added protection and to decrease movement of the dressings. During recovery, pigs were given an intramuscular injection of $0.1 \mathrm{mg} / \mathrm{kg}$ buprenorphine.

Animals were observed daily for wound care, pain management, and evidence of infection. Donor-site dressings were changed only if needed due to shifting or saturation, while external dressings were replaced daily. Opsite was replaced on postoperative day 3 in the 5-day animal. On day 3 or 5, pigs were euthanized, wounds were examined for infection, and full-thickness samples (including 0.5-cm margin of unwounded skin surrounding each wound) were excised from the center of each wound and processed for histology (Fig. 2).

For each dressing material, ease of use (which included both application and wound care) was

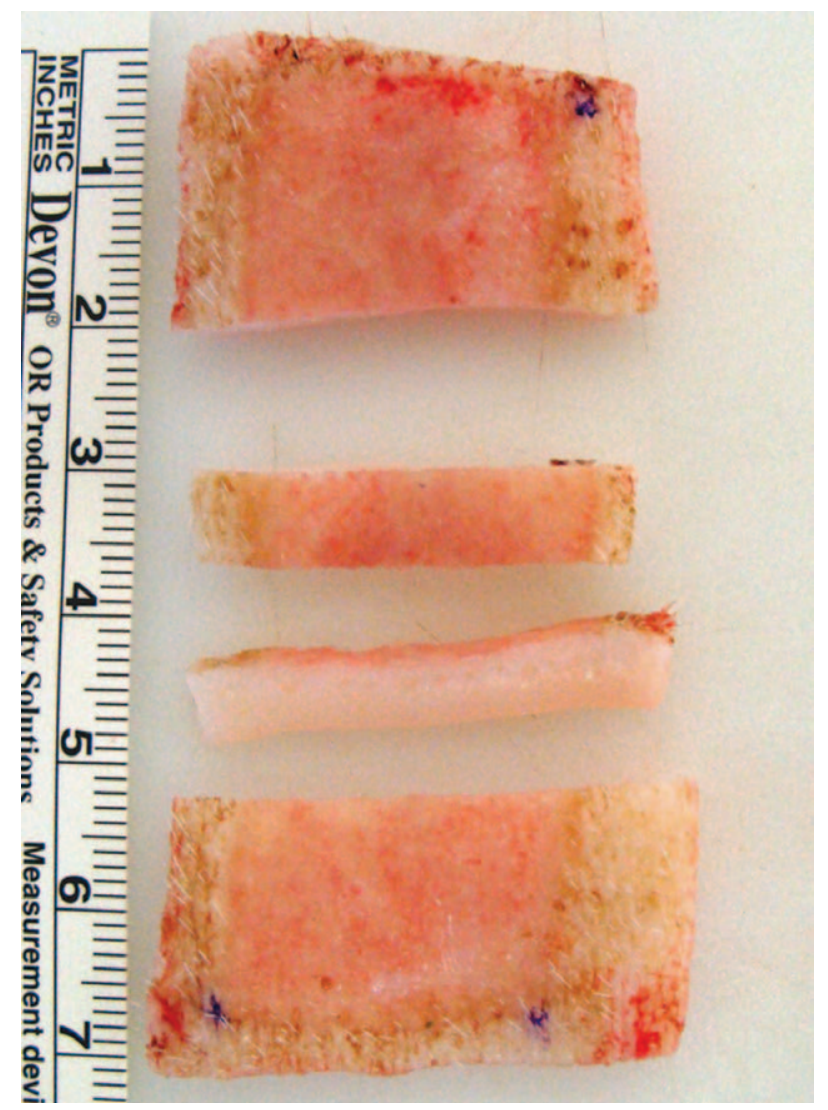

Fig. 2. Full-thickness explant harvested on postoperative day 3 , including $0.5-\mathrm{cm}$ margin of unwounded skin surrounding the $4 \times 2 \mathrm{~cm}$ partial-thickness wound. Two $0.5-\mathrm{cm}$-thick specimens were excised from the center of each wound and processed for histology. The lower middle piece is turned on its side to view the epidermis, dermis, and subcutaneous fat. 
scored by 3 investigators directly involved in the pigs' wound care during the course of the study. An overall score was given which ranged from 1 (useless due to difficulty) to 10 (the easiest use ever possible). Although subjective, factors considered by the observers in the ease of use score included ease of application, ease of removal, dressing adherence, leakage, and frequency of dressing changes.

\section{Histology and Morphometric Analysis}

Tissues were fixed 72 hours in $10 \%$ neutral buffered formalin, embedded in paraffin, and $5 \mu \mathrm{m}$ serial sections were stained with hematoxylin-eosin, Masson trichrome, and elastic van Gieson. Digital images were captured using PictureFrame software (Optronics, Goleta, Calif.) and an Olympus IX71 microscope (Olympus America, Center Valley, $\mathrm{Pa}$.). Sections stained with hematoxylin-eosin were analyzed at $100 \times$ magnification by a single experienced blinded observer (L.M.P.) for the amount of new epithelium in each wound, which was defined as the region between the wound margins covered by epithelial cell islets and cells growing out of regenerating hair follicles. To ensure that differences in wound sizes were taken into account, reepithelialization was standardized by dividing the new epithelium by the wound length in each section to calculate the percent reepithelialization. The thickness of the new epithelium in each wound was measured in a minimum of 5 fields (5-10 measurements per field yielding 25-50 measurements per wound) at $200 \times$ magnification using the PictureFrame application calibrated with $50-\mu \mathrm{m}$ glass beads.

Total nuclei were counted by a single experienced blinded observer in 3 randomly selected high power fields (HPFs) per section at $1000 \times$ magnification (similar to Mauskar et $\mathrm{al}^{20}$ ). All tissues were aligned so that the viable dermis was located at the top of the field. Acute inflammatory cells (polymorphonuclear leukocytes, PMN) were identified morphologically, counted, and semiquantitatively scored as $1=<10 \mathrm{PMN}$ per $\mathrm{HPF}, 2=10-20 \mathrm{PMN}$ per $\mathrm{HPF}$, or $3=>20$ per HPF. Inflammation was also subjectively scored on a scale of $0-4(0=$ none, $1=$ minimal, $2=$ mild, $3=$ moderate, and $4=$ severe , and sections were evaluated qualitatively for cell types present and granuloma tissue formation.

\section{Immunohistochemistry}

Immunohistochemistry was performed on $5 \mu \mathrm{m}$ paraffin sections from 6 wounds and unwounded skin from each of the 4 pigs using a mouse monoclonal antibody to pan cytokeratin (C-11, ab7753,
Abcam, Cambridge, Mass.) to confirm identification of newly formed epithelium. After deparaffinization and rehydration, heat-mediated antigen retrieval with citrate buffer (Millipore, Temecula, Calif.) was performed, and immunostaining was accomplished with the use of the Dako EnVision System (Dako North America, Carpinteria, Calif.) following the manufacturer's instructions. Briefly, sections were incubated with an endogenous enzyme block solution for 5 minutes, incubated for 15 minutes with the primary antibody applied at a dilution of 1:250, and then incubated for 25 minutes with a dextran polymer conjugated with horseradish peroxidase and affinity-isolated immunoglobulins. Rinsed sections were incubated for 10 minutes with a 3,3'-diaminobenzidine chromogen-substrate to form a colored reaction product. Slides were counterstained with Mayer's hematoxylin (Dako North America), dehydrated, and permanently mounted. Negative controls were achieved by omission of the primary antibody and by applying a mouse universal negative control reagent (Dako North America) instead of the mouse monoclonal primary antibody.

\section{Statistical Analysis}

Three pigs were evaluated at 3 days and 1 pig was evaluated at 5 days ( 4 wounds per dressing type per pig). Reepithelialization data from the 3 independent pigs at 3 days were pooled to give a sample of 12 wounds per dressing type at 3 days. Pooled analyses are commonly performed in partial-thickness wounding studies using 2 or 3 pigs per group. ${ }^{21-23}$ Epithelial thickness data were collected for each wound (25-50 measurements per wound), and the mean of 4 wounds per dressing type was calculated for each pig. The epithelial thickness means from the 3 animals at 3 days were then averaged yielding a sample of 3 wounds per dressing type at 3 days. Total cell nuclei and acute inflammatory cells were counted in 3 HPFs per wound from 2 wounds per dressing type per pig, giving a total sample of 18 HPFs per dressing type from the 3 pigs at 3 days and a sample of 6 HPFs per dressing type from 1 pig at 5 days. Values were expressed as mean \pm SEM. Analysis of variance was used to determine differences among experimental groups followed by post hoc pairwise multiple comparisons using the Holm-Sidak method to identify specific differences between the groups. Comparisons between 2 dressings were performed using the $t$ test. Corresponding nonparametric tests were used when indicated. Statistical analyses were performed using SigmaPlot 11.2 software (Systat Software, San Jose, Calif.), with $P<0.05$ considered significant. 


\section{RESULTS}

\section{Wound Care and Macroscopic Findings}

The procedure was well tolerated in all animals with no adverse effects or visible signs of pain. None of the skin graft donor sites became infected. Although some interanimal variability occurred, gross inspection of wounds on days 3 and 5 revealed that Xeroform- and DuoDERM-treated wounds were consistently among the best healed sites, with complete or nearly complete healing observed on day 5 (Fig. 3). Opsite-treated sites retained the most moisture, while Kaltostat and Aquacel were most adherent during removal. Dressings differed with respect to ease of use $(P=0.03)$, with Mepilex and Xeroform receiving the highest scores (Table 1 ). These 2 dressings were easy to apply and were very rarely (Xeroform) or never (Mepilex) replaced or adjusted during the postoperative period up to 5 days. Although Mepilex required no dressing changes throughout the duration of the study, Xeroform was changed on one occasion due to shifting when one animal was able to remove all outer dressing materials. By contrast, Aquacel and Kaltostat required frequent reinforcement due to shifting and thus required dressing changes daily. Opsite was prone to leakage due to its moisture-retaining properties and shifted in highly exudative wounds. Because of these factors, all Opsite dressings were changed on day 3 in the 5-day animal. DuoDERM stayed in place through- out the duration of the study and most wounds did not require changing throughout the experiment (only one DuoDERM-treated wound in one animal needed to be changed due to shifting). Cost analyses revealed that Xeroform was most cost-effective and Aquacel was most expensive (Table 1).

\section{Microscopic Findings}

Because comparable epithelial morphology was observed from the hematoxylin-eosin, Masson trichrome, elastic van Gieson, and pan cytokeratin staining, we chose to present histological results from hematoxylin-eosin-stained sections, similar to other published studies using pigs. ${ }^{20-23}$

On day 3, all wounds showed evidence of reepithelialization, although differences were observed among the various dressings (Figs. 4 and 5). Within each pig, a statistically significant difference among treatments was observed (pig 1, $P=0.002$; pig 2, $P=0.04$; pig 3, $P<0.001)$. When comparing Xeroform to the other dressings evaluated in this study, post hoc analyses revealed that Xeroform performed better than Mepilex, Kaltostat, and Aquacel in pig 1, but less well than DuoDERM and Kaltostat in pig 3. No statistically significant difference between Xeroform and the other dressings was observed in pig 2. Pooled analysis at 3 days ( $n=12$ wounds per dressing type) revealed a statistically significant difference among dressings $(P<0.001)$, with DuoDERM-treated wounds having a faster reepithelialization rate and Mepilex-treated wounds having a reduced reepithelialization rate
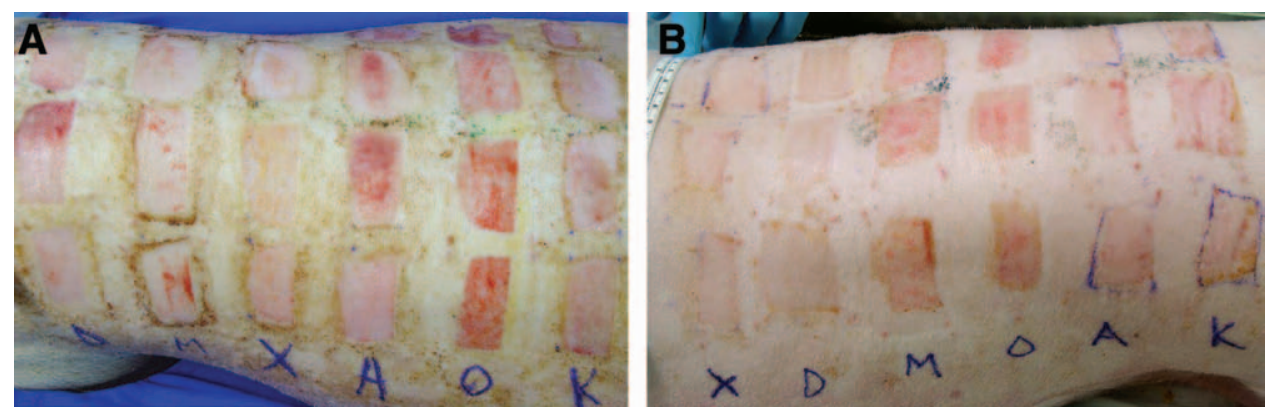

Fig. 3. Split-thickness wound appearance on day 3 (A) and day 5 (B) after surgery. Most wounds have completely or nearly completely reepithelialized by $5 \mathrm{~d}$.

Table 1. Incidence of Infection, Ease of Use, and Cost Comparisons among Dressing Materials

\begin{tabular}{lccc}
\hline Dressing Material & Incidence of Infection & Ease of Use $^{*}(\mathbf{m e a n} \pm \mathbf{S E M})$ & Cost $_{\left(\mathbf{c e n t s} / \mathbf{c m}^{2}\right)}$ \\
\hline Xeroform & None & $8.0 \pm 0$ & 0.17 \\
Opsite & None & $5.3 \pm 0.7^{\dagger}$ & 0.88 \\
DuoDERM & None & $7.7 \pm 0.3$ & 1.48 \\
Mepilex & None & $9.3 \pm 0.3^{\dagger}$ & 3.37 \\
Kaltostat & None & $5.0 \pm 1.5$ & 3.50 \\
Aquacel & None & $5.0 \pm 1.5$ & 7.10 \\
\hline
\end{tabular}

*Ease of use included both application and wound care and was scored between 1 (useless due to difficulty) and 10 (the easiest use ever possible).

${ }^{+} P<0.05$ vs Xeroform, $t$ test. 
PRS GO・ 2014
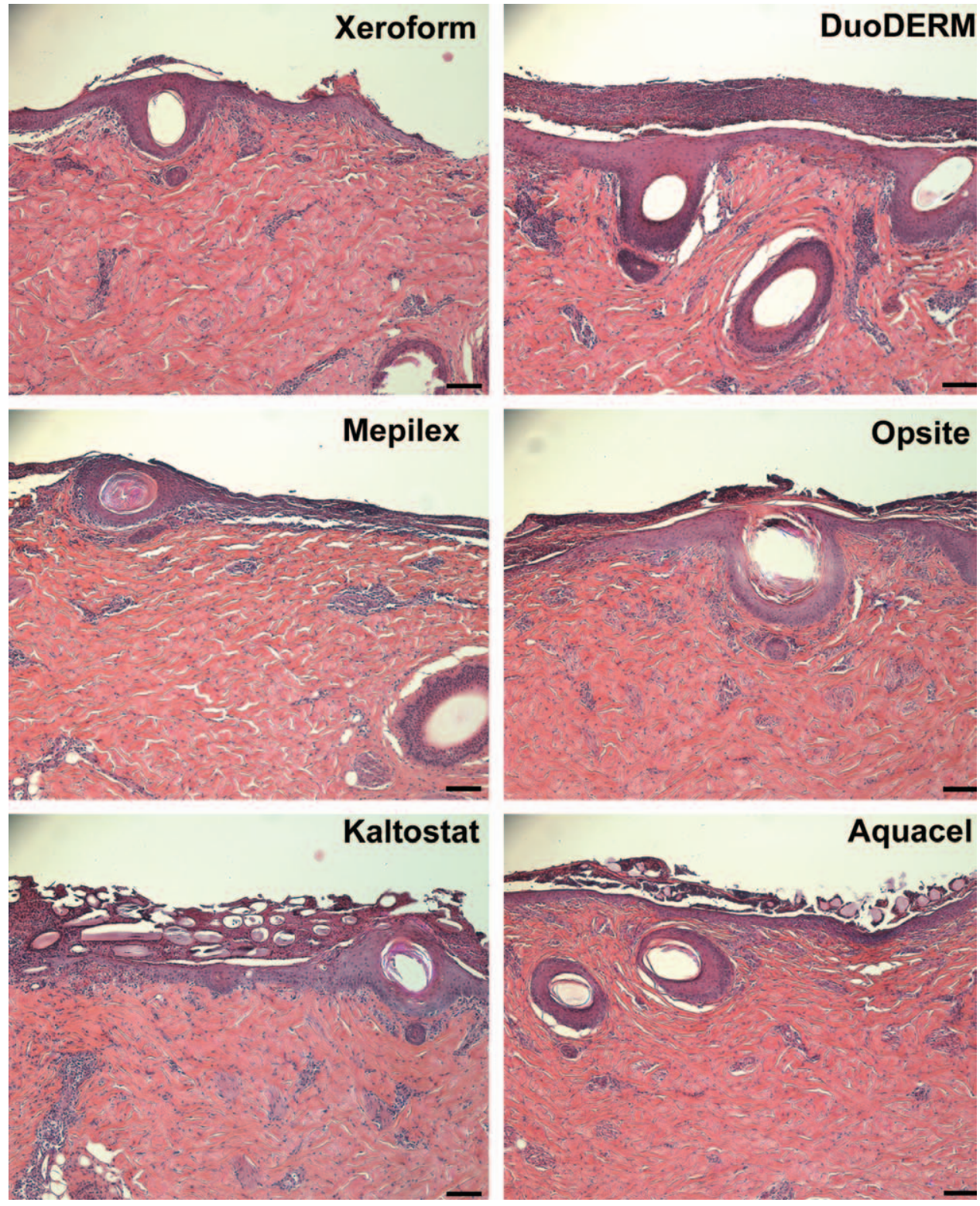

Fig. 4. Representative hematoxylin-eosin-stained sections of wounds $3 \mathrm{~d}$ after treatment. Mepilex-treated wounds showed less epithelial migration extending outward from hair follicles than the other dressings. A mixture of fibrin clot, dead polymorphonuclear leukocytes, and degraded extracellular matrix components at the interface between the dressings and the healthy underlying dermis can be visualized sloughing off after epidermis formation. Note Kaltostat and Aquacel fibers surrounded by the mixture of the dermis-clot interface. Scale bar $=100 \mu \mathrm{m}$.

compared to Xeroform (Fig. 5). Reepithelialization in Xeroform-treated wounds was not significantly different than in wounds treated with Opsite, Kaltostat, and Aquacel (Fig. 5). Mepilex-treated wounds overall demonstrated the least reepithelialization outside of regions immediately adjacent to hair follicles, while DuoDERM demonstrated the greatest $(P=0.004$, $81.3 \% \pm 7.2 \%$ DuoDERM vs $32.9 \% \pm 4.2 \%$ Mepilex, $t$ test, $n=3$ at 3 days). In some areas treated with each dressing type at 3 days, the epithelium was im- mature in appearance and separation of the newly formed epithelium from the underlying dermis was observed (not shown). DuoDERM, however, consistently demonstrated the most mature looking epithelium with minimal dermal-epidermal separation. At 5 days, wounds treated with Xeroform, DuoDERM, and Kaltostat showed $100 \%$ reepithelialization, while those treated with Aquacel $(99.3 \% \pm 0.7 \%)$ and Mepilex $(85.5 \% \pm 4.5 \%)$ were nearly completely reepithelialized. 


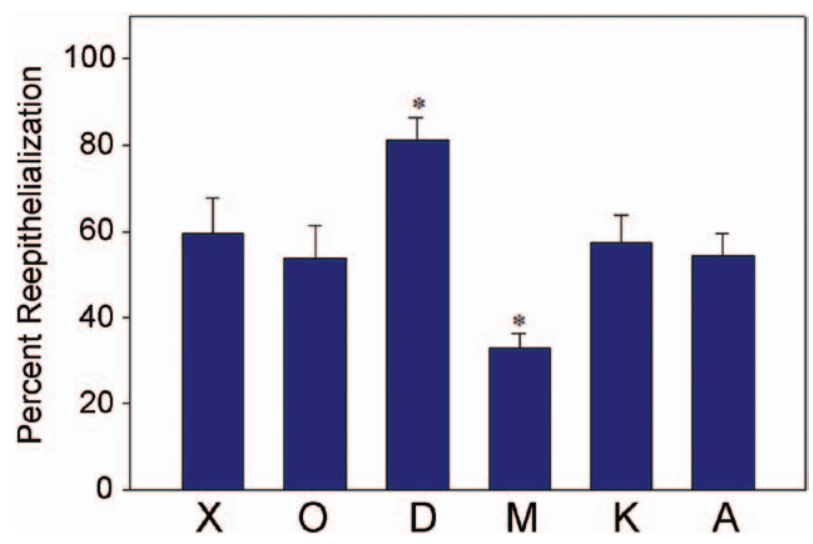

Fig. 5. Percent reepithelialization $3 \mathrm{~d}$ after wounding. Values are reported as the mean \pm SEM of 12 wounds per dressing type. A statistically significant difference among dressings was observed ${ }^{*} P<0.05$ compared to Xeroform, analysis of variance followed by multiple comparisons vs Xeroform using the Holm-Sidak method). X, Xeroform; O, Opsite; D, DuoDERM; M, Mepilex; K, Kaltostat; A, Aquacel.

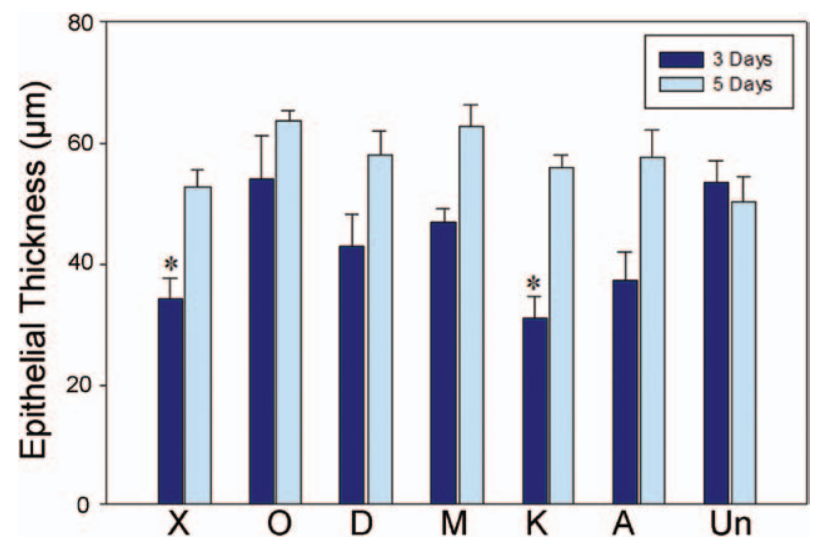

Fig. 6. Epithelial thickness $3 d(n=3$ pigs) and $5 d(n=1$ pig) after wounding. At $3 \mathrm{~d}$, new epithelium was thinner than that from unwounded skin for both Xeroform- and Kaltostat-treated wounds ( ${ }^{*} P<0.05$ compared to unwounded skin, analysis of variance followed by multiple comparisons vs unwounded skin using the Holm-Sidak method). At $5 \mathrm{~d}$, epithelial thickness following any treatment was not different from unwounded skin $(P=0.11)$. Un, unwounded skin.
A significant difference in epithelial thickness was noted among dressings at 3 days $(P=0.04)$ but not at 5 days $(\mathrm{p}=0.23)$. At 3 days, new epithelium was thinner than that from unwounded skin for both Xeroformand Kaltostat-treated wounds ( $P=0.02$; Fig. 6$)$. Thickness of new epithelium for all treatments increased to that of unwounded skin by 5 days $(P=0.11)$.

Inflammatory response was examined as an indicator of the relative biocompatibility of each dressing type. All treatments showed minimal to mild inflammation at 3 days [subjective inflammation scores ranged from $1.2 \pm 0.18$ (Xeroform) to $1.75 \pm 0.19$ (Opsite), $P=0.47$ ], with inflammatory cells consisting predominantly of PMN, lymphocytes, and macrophages. All wounds demonstrated increased inflammation relative to unwounded skin at both 3 and 5 days $(P<0.001)$. Number of total nuclei (cellularity) was not significantly different among dressing types at 3 days $(P=0.07)$ or 5 days $(P=0.42$; Table 2$)$. Although cellularity increased in day 5 wounds compared to day 3 wounds for all dressings, presumably due to the increase in fibroblasts and inflammatory cells such as macrophages, this increase reached statistical significance for Xeroform, Opsite, and Mepilex (Table 2). PMN score was not statistically different among the dressing types at 3 days $(P=0.06)$ or 5 days $(P=1.00)$, although Xeroform and Mepilex tended to have the lowest scores on day 3 (Table 2). PMN score decreased in day 5 wounds compared to day 3 wounds, but this decrease reached statistical significance only for Opsite, DuoDerm, Kaltostat, and Aquacel (Table 2).

Each dressing elicited relatively low numbers of lymphocytes, indicating that none of the dressings produced a pathologically enhanced immune response beyond that of normal wound healing. A mixture of fibrin clot, dead PMN, and degraded extracellular matrix components at the interface between the dressings and the healthy underlying dermis was observed to slough off after epidermis formation (Figs. 4 and 7). By 5 days for each dressing

Table 2. Acute Inflammatory Cell (PMN) Scores and Density of Nuclei (Cellularity) per HPF at 1000x Magnification

\begin{tabular}{|c|c|c|c|c|c|c|c|c|c|}
\hline Parameter & Days & Xeroform & Opsite & DuoDERM & Mepilex & Kaltostat & Aquacel & $\begin{array}{c}\text { (3- and 5-Day Animals } \\
\text { Combined) }\end{array}$ & $P^{*}$ \\
\hline PMN Score & 3 & $1.44(0.15)$ & $1.90(0.20)$ & $2.10(0.19)$ & $1.50(0.19)$ & $2.11(0.20)$ & $1.84(0.21)$ & $1.00(0)$ & 0.06 \\
\hline PMN Score & 5 & $1.00(0)$ & $1.00(0)^{\dagger}$ & $1.00(0)^{\dagger}$ & $1.00(0)$ & $1.00(0)^{\dagger}$ & $1.00(0)^{\dagger}$ & $1.00(0)$ & 1.00 \\
\hline Cellularity & 3 & $75(5)$ & $86(7)$ & $95(7)$ & $74(5)$ & $93(5)$ & $94(9)$ & $62(5)$ & 0.07 \\
\hline Cellularity & 5 & $112(12)^{\dagger}$ & $127(6)^{\dagger}$ & 104 (12) & $120(9)^{\dagger}$ & $102(10)$ & $111(6)$ & $62(5)$ & 0.42 \\
\hline
\end{tabular}

For the analysis at $3 \mathrm{~d}$, cells were counted in 18 HPFs per dressing type from a total of 3 pigs (3 HPF analyzed per wound, 2 wounds per dressing type per pig). Cells were counted in a total of 6 HPF per dressing type from 1 pig at $5 \mathrm{~d}$ ( $3 \mathrm{HPF}$ analyzed per wound, 2 wounds per dressing type per pig). Data are presented as mean (SEM). PMN score: $1=<10$ PMN per HPF, $2=10-20$ PMN per HPF, and $3=>20$ PMN per HPF. *Analysis of variance comparing the 6 dressing materials.

${ }^{\dagger} P<0.05$ vs $3 \mathrm{~d}, t$ test. 
PRS GO・ 2014
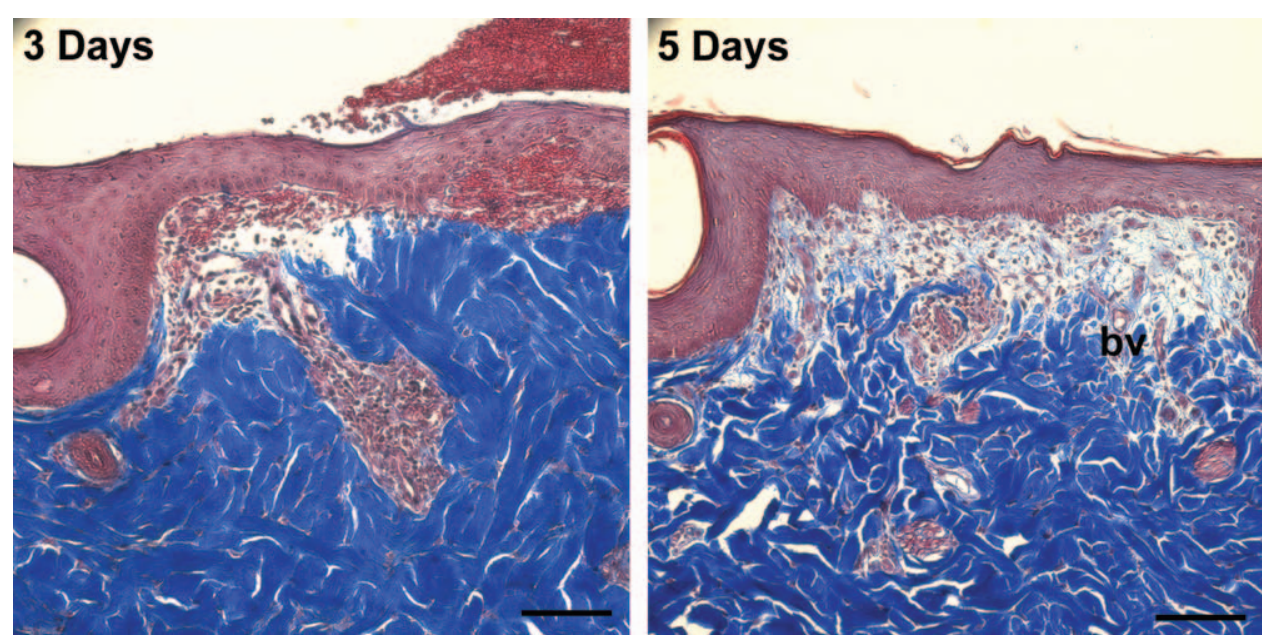

Fig. 7. Representative Masson trichrome-stained sections of wounds treated with DuoDERM on days 3 and 5 after surgery demonstrating granuloma tissue formation and tissue remodeling. By $5 \mathrm{~d}$, the number of inflammatory cells has decreased and the number of fibroblasts has increased, with new collagen (light blue) and blood vessel (bv) formation visible. In addition, the epithelium is maturing with stratum corneum observed. Scale bar $=100 \mu \mathrm{m}$.

type, the number of inflammatory cells (particularly PMN) decreased and the number of fibroblasts increased, with new collagen and blood vessel formation observed indicative of granuloma tissue formation and tissue remodeling (Fig. 7 and Table 2).

Overall Performance Ranking of Dressings for the Management of Split-thickness Skin Graft Donor Sites

A scoring system integrating the major parameters studied (percent reepithelialization, inflammation, infection, ease of use, and cost) was used to rank the overall performance of the donor-site dressings. Unlike human studies that administer questionnaires to patients, pain or discomfort was unable to be assessed in this animal study and therefore could not be included in the overall ranking of performance. For each of the parameters, 3 stars were assigned to the best performance, 2 stars to the moderate performance, and 1 star to the weakest performance (Table 3). Combined scoring revealed that DuoDERM and Xeroform were tied for the highest rank. While DuoDERM exhibited the best score for reepithelialization, Xeroform had the added benefit of cost. Opsite lost a point for ease of use, and
Mepilex lost points for reepithelialization and cost. Kaltostat and Aquacel, although receiving similar scores for reepithelialization compared to Xeroform and Opsite, lost points for cost and ease of use. Distinctive aspects of each dressing material compiled from the findings of this study are listed below and in Table 3 and are ranked in decreasing order of the total score.

\section{DuoDERM (Hydrocolloid)}

This dressing maintains moisture and thus is prone to leakage and/or shifting and may need to be changed in highly exudative wounds, although overwhelming exudate occurred infrequently in our study. Additionally, because of the moisture-preserving properties, this dressing on occasion forms a thick paste-like drainage which may be concerning to uninformed patients leading to potential clinic visits. DuoDERM does not require an overlying dressing and stayed in place well unless the wound was highly exudative; overall this is a low maintenance dressing in terms of nursing care, but moderate in cost. This dressing received the highest scores for reepithelialization.

Table 3. Ranking of Dressing Materials According to Total Score

\begin{tabular}{lcccccc}
\hline Dressing Material & $\begin{array}{c}\text { 3-Day \% Reepi- } \\
\text { thelialization }\end{array}$ & Inflammation & $\begin{array}{c}\text { Incidence of } \\
\text { Infection }\end{array}$ & Ease of Use & Cost & Total Score \\
\hline DuoDERM & $* * *$ & $* * *$ & $* * *$ & $* *$ & $* *$ & 13 \\
Xeroform & $* *$ & $* * *$ & $* * *$ & $* *$ & $* *$ & 13 \\
Opsite & $* *$ & $* * *$ & $* * *$ & $* * *$ & $* *$ & 12 \\
Mepilex & $*$ & $* * *$ & $* * *$ & $*$ & $* *$ & 11 \\
Kaltostat & $* *$ & $* * *$ & $* * *$ & $*$ & $*$ \\
Aquacel & $* *$ & $* * *$ & $* * *$ & \\
\hline
\end{tabular}

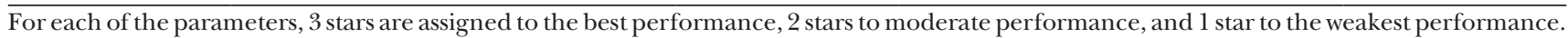




\section{Xeroform (Fine-mesh Gauze Impregnated with the Antiseptic Bismuth Tribromophenate)}

This dressing does require an overlying gauze dressing in human subjects, but the overlying dressing only needs to be changed if it becomes saturated. Xeroform is intended to stay in place throughout the healing process and fall off on its own when epithelialization is complete. This dressing requires little to no additional nursing care, and in our study, this dressing was changed only on one occasion due to shifting when one animal was able to remove all outer dressing materials. Xeroform is the most economical of the 6 dressings evaluated in this study.

\section{Opsite (Polyurethane Film)}

Although more difficult to apply, this dressing does not require an overlying dressing and overall stays in place well; however, it requires increased nursing care (it required a dressing change on day 3 during our study unlike DuoDERM, Xeroform, and Mepilex). Like DuoDERM, because of its moisture-retaining properties, Opsite is prone to leakage and shifting in highly exudative wounds. Opsite has the added benefit of cost, although this dressing may become less economical if the frequency of dressing changes is increased.

\section{Mepilex (Silicone-faced Polyurethane Foam)}

In human use, this dressing would likely not require an overlying dressing due to its absorbent properties. Mepilex did not require any dressing changes and stayed in place throughout the duration of our study. It was easily removed at the conclusion of the study. This dressing should require little to no additional nursing care once applied. Mepilex, however, received the lowest scores for reepithelialization of the 6 dressings evaluated in this study, which may offset the benefits of reduced nursing care and may not justify its increased cost.

\section{Kaltostat (Calcium Alginate) and Aquacel (Hydrofiber)}

These 2 dressings were similarly high maintenance in terms of dressing care in our study. In all wounds, these dressings were frequently prone to shifting requiring dressing changes daily. Kaltostat and Aquacel both require an overlying nonadherent dressing. Additionally, there is potential for increased clinic visits due to patient concerns over their tendency to form a gel-like substance overlying the wound. In our study, we found that the increased expense of Kaltostat and Aquacel and increased nursing care requirements preclude their routine use for split-thickness skin graft donor sites.

\section{DISCUSSION}

Donor-site dressing materials are broadly classified as moist and nonmoist, varying in their biochemical composition and absorptive capabilities. ${ }^{10}$ Although a moist wound environment has been suggested to increase reepithelialization, decrease patient discomfort, and avoid damage to newly formed epithelium when the dressing is removed, ${ }^{4-7,17,18,24}$ there is no clear evidence to support the claim of overall superiority of moist over nonmoist dressings. ${ }^{10}$ In this study, we compared the effectiveness of 5 commonly used moist dressings with our standard dressing, Xeroform (a nonmoist dressing), and showed that DuoDERM and Xeroform were the most effective dressings overall. Because none of the wounds became infected, and all dressings elicited minimal to mild inflammation, the distinguishing parameters used to rank the dressings were reepithelialization, ease of use (application and wound care), and cost-effectiveness.

Aggregated results revealed that hydrocolloids such as DuoDERM demonstrate superior reepithelialization, increased patient comfort, and comparable infection rates relative to other materials. ${ }^{7,18}$ DuoDERM is composed of an outer layer of hydrocolloid polymer complex which is both occlusive and hydrophilic, aiding removal of excess exudates while maintaining an environment thought to facilitate increased collagen synthesis, keratinocyte migration, and angiogenesis. ${ }^{6,7,11,24}$ A recent clinical trial showed that time to complete reepithelialization was 7 days shorter with the use of DuoDERM than with alginate, polyurethane film, impregnated gauze, hydrofiber, or silicone. ${ }^{8}$ Similarly, $97 \%$ of wounds treated with DuoDERM were completely healed by 10 days compared with $75 \%$ of wounds treated with the hydrogel Zenoderm. ${ }^{25}$ In addition, donor areas treated with hydrocolloids had a smoother, more even appearance when compared to sites treated with calcium alginate dressings. ${ }^{26}$ Consistent with previous clinical studies, ${ }^{6-8,25,27}$ we showed that DuoDERM elicited the fastest rate of healing while being moderate in price, easy to use, and resistant to infection.

We found Xeroform to be easy to use, resistant to infection, cost-effective, and only slightly inferior to DuoDERM in terms of reepithelialization, suggesting that Xeroform should remain a favored choice for use on split-thickness skin graft donor sites in cases in which time to reepithelialization is not of utmost importance. While maintaining rapid healing times, Xeroform is nearly 9 times less expensive than DuoDERM. Although the authors of a recent clinical trial advised discontinuation of gauze dressings due to increased infections, they investigated Adaptic petrolatum gauze (Systagenix, Gatwick, UK) 
and paraffin gauze, but not Xeroform. ${ }^{8}$ Aggregated results of gauze dressings for donor-site wounds did not find an increased risk of infection. ${ }^{7,10,17,18}$

We did not see an overall improvement in reepithelialization of wounds treated with Kaltostat, Aquacel, or Opsite when compared to those treated with Xeroform. Although national surveys revealed that Kaltostat is the most commonly used dressing in the United Kingdom and Australia, ${ }^{3,16}$ clinical data favor other dressing types in terms of wound healing, cost, patient comfort, and ease of use. ${ }^{17,28-30}$ We and others ${ }^{13,30}$ found Aquacel and Kaltostat to be expensive and relatively difficult to use, as these dressings required frequent reinforcement, thus precluding their routine use for split-thickness skin graft donor sites. Although Opsite provided rapid reepithelialization at a relatively low cost, we found it difficult to apply, with frequent shifting of the dressing when high exudate load existed, similar to observations by Demirtas et al. ${ }^{6}$ Finally, although Mepilex was the easiest to use, not requiring any dressing changes during the postoperative period or adjustments due to shifting, Mepilex-treated wounds elicited the least reepithelialization overall. Given its reduced wound healing properties and moderate cost, we and oth$\mathrm{ers}^{31}$ determined that Mepilex is inferior for use on split-thickness donor sites compared to other dressings evaluated in this study.

Our study had limitations. Although pigs are among the best preclinical models available for comparison with human skin, some inherent physiologic differences exist, including faster wound healing in pigs than humans. ${ }^{32,33}$ The use of pigs did not enable the evaluation of pain in this study; however, the animals did not show signs of discomfort during the postoperative period. Overall pain scores have been quite low in clinical trials and differences between dressings minor, suggesting that the clinical relevance of differences in pain among dressings is questionable. ${ }^{8,13,31}$ In addition, this short-term study did not enable the evaluation of scar appearance. Because previous studies have demonstrated no long-term differences in scar quality, the clinical relevance of this shortcoming is undetermined. ${ }^{6,13,31}$ Finally, despite a small sample size, the use of 3 pigs and 12 wounds per dressing type was sufficient to detect some important differences among dressings on the third postoperative day.

\section{CONCLUSIONS}

Ideal wound care for split-thickness skin graft donor sites should include dressings that promote healing, prevent complications, and are cost-effective. An objective comparison of 6 commonly used dressing materials evaluated in a swine model revealed that DuoDERM and Xeroform were most effective overall. DuoDERM tended to outperform all dressings in reepithelialization at 3 days, whereas Xeroform was least expensive, easy to use, and demonstrated rapid reepithelialization. These findings suggest that Xeroform may be preferred for use on large donor-site areas. DuoDERM may be more appropriate for use on small donor sites when time to healing is a priority.

Lisa M. Pierce, DSc

Department of Clinical Investigation Tripler Army Medical Center 1 Jarrett White Road, Honolulu HI 96859-5000 E-mail: lisa.m.pierce.civ@mail.mil

1. Ratner D. Skin grafting. Semin Cutan Med Surg. 2003;22:295-305.

2. McCain D, Sutherland S. Nursing essentials: skin grafts for patients with burns. Am J Nurs. 1998;98:34-38; quiz 39.

3. Geary PM, Tiernan E. Management of split skin graft donor sites-results of a national survey. J Plast Reconstr Aesthet Surg. 2009;62:1677-1683.

4. Fernandes de Carvalho V, Paggiaro AO, Isaac C, et al. Clinical trial comparing 3 different wound dressings for the management of partial-thickness skin graft donor sites. J Wound Ostomy Continence Nurs. 2011;38:643-647.

5. Argirova M, Hadjiski O, Victorova A. Acticoat versus Allevyn as a split-thickness skin graft donor-site dressing: a prospective comparative study. Ann Plast Surg. 2007;59:415-422.

6. Demirtas Y, Yagmur C, Soylemez F, et al. Management of split-thickness skin graft donor site: a prospective clinical trial for comparison of five different dressing materials. Burns 2010;36:999-1005.

7. Wiechula R. The use of moist wound-healing dressings in the management of split-thickness skin graft donor sites: a systematic review. Int J Nurs Pract. 2003;9:S9-S17.

8. Brölmann FE, Eskes AM, Goslings JC, et al; REMBRANDT study group. Randomized clinical trial of donor-site wound dressings after split-skin grafting. Br J Surg. 2013;100:619-627.

9. Barnea Y, Amir A, Leshem D, et al. Clinical comparative study of aquacel and paraffin gauze dressing for split-skin donor site treatment. Ann Plast Surg. 2004;53:132-136.

10. Voineskos SH, Ayeni OA, McKnight L, et al. Systematic review of skin graft donor-site dressings. Plast Reconstr Surg. 2009;124:298-306.

11. Feldman DL, Rogers A, Karpinski RH. A prospective trial comparing Biobrane, Duoderm and xeroform for skin graft donor sites. Surg Gynecol Obstet. 1991;173:1-5.

12. Kaiser D, Hafner J, Mayer D, et al. Alginate dressing and polyurethane film versus paraffin gauze in the treatment of split-thickness skin graft donor sites: a randomized controlled pilot study. Adv Skin Wound Care 2013;26:67-73.

13. Dornseifer U, Lonic D, Gerstung TI, et al. The ideal split-thickness skin graft donor-site dressing: a clinical comparative trial of a modified polyurethane dressing and aquacel. Plast Reconstr Surg. 2011;128:918-924.

14. Persson K, Salemark L. How to dress donor sites of split thickness skin grafts: a prospective, randomised study 
of four dressings. Scand J Plast Reconstr Surg Hand Surg. 2000;34:55-59.

15. Hermans MH. Results of an internet survey on the treatment of partial thickness burns, full thickness burns, and donor sites. J Burn Care Res. 2007;28:835-847.

16. Lyall PW, Sinclair SW. Australasian survey of split skin graft donor site dressings. Aust N Z J Surg. 2000;70:114-116.

17. Rakel BA, Bermel MA, Abbott LI, et al. Split-thickness skin graft donor site care: a quantitative synthesis of the research. Appl Nurs Res. 1998;11:174-182.

18. Schreuder S, Qureshi M, Vermeulen H, et al. Dressings and topical agents for treating donor sites of split-skin grafts: a systematic review. EWMA J. 2009;9:22.

19. Sullivan TP, Eaglstein WH, Davis SC, et al. The pig as a model for human wound healing. Wound Repair Regen. 2001;9:66-76.

20. Mauskar NA, Sood S, Travis TE, et al. Donor site healing dynamics: molecular, histological, and noninvasive imaging assessment in a porcine model. J Burn Care Res. 2013;34:549-562.

21. Kleinbeck KR, Faucher L, Kao WJ. Multifunctional in situ photopolymerized semi-interpenetrating network system is an effective donor site dressing: a cross comparison study in a swine model. J Burn Care Res. 2009;30:37-45.

22. Kleinbeck KR, Faucher LD, Kao WJ. Biomaterials modulate interleukin- 8 and other inflammatory proteins during reepithelialization in cutaneous partial-thickness wounds in pigs. Wound Repair Regen. 2010;18:486-498.

23. Peura M, Kaartinen I, Suomela S, et al. Improved skin wound epithelialization by topical delivery of soluble factors from fibroblast aggregates. Burns 2012;38:541-550.

24. Winter GD. Formation of the scab and the rate of epithelialization of superficial wounds in the skin of the young domestic pig. Nature 1962;193:293-294.
25. Tan ST, Roberts RH, Sinclair SW. A comparison of Zenoderm with DuoDERM E in the treatment of split skin graft donor sites. Br J Plast Surg. 1993;46:82-84.

26. Porter JM. A comparative investigation of re-epithelialisation of split skin graft donor areas after application of hydrocolloid and alginate dressings. Br J Plast Surg. 1991;44:333-337.

27. Solanki NS, Mackie IP, Greenwood JE. A randomized prospective study of split skin graft donor site dressings: AWBAT-D vs. Duoderm. Burns 2012;38:889-898.

28. Terrill PJ, Goh RC, Bailey MJ. Split-thickness skin graft donor sites: a comparative study of two absorbent dressings. J Wound Care 2007;16:433-438.

29. Vaingankar NV, Sylaidis P, Eagling V, et al. Comparison of hydrocellular foam and calcium alginate in the healing and comfort of split-thickness skin-graft donor sites. J Wound Care 2001;10:289-291.

30. Hormbrey E, Pandya A, Giele H. Adhesive retention dressings are more comfortable than alginate dressings on split-skin-graft donor sites. Br J Plast Surg. 2003;56: 498-503.

31. Kaartinen IS, Kuokkanen HO. Suprathel(®) causes less bleeding and scarring than Mepilex $\left({ }^{\circledR}\right)$ Transfer in the treatment of donor sites of split-thickness skin grafts. J Plast Surg Hand Surg. 2011;45:200-203.

32. Genecov DG, Schneider AM, Morykwas MJ, et al. A controlled subatmospheric pressure dressing increases the rate of skin graft donor site reepithelialization. Ann Plast Surg. 1998;40:219-225.

33. Ward C, Ciraulo D, Coulter M, et al. Does treatment of split-thickness skin grafts with negative-pressure wound therapy improve tissue markers of wound healing in a porcine experimental model? J Trauma Acute Care Surg. 2012;73:447-451. 